\title{
More than half of dentists say stress is affecting their practice
}

Almost half of dentists say stress in their job is exceeding their ability to cope and the most stressful aspects of their work are related to regulation and fear of litigation from patients, according to a new large-scale study published in this issue of $B D J$.

Researchers from the BDA found high levels of stress and burnout amongst a survey of more than 2,000 UK dentists, amongst whom almost a fifth (17.6\%) admitted they had seriously thought about committing suicide.

There is existing evidence that dentistry is a stressful profession mostly due to the nature and working conditions in the dental surgery and although there has been work done on occupational stress in dentistry, there has been far less investigation of the psychological distress of the job and what impact that has on dentists' well-being and the care they provide to patients.

Therefore a team of researchers from the BDA set out to determine the levels of stress and burnout in UK dentists and how this related to their well-being while also identifying the sources of work-related stress that dentists reported in different fields of practice.

Using an online survey of BDA members and non-members, they analysed responses from 2,053 dentists.

A questionnaire was constructed, covering various areas including demographics; working practices; assessments of mental health, health and well-being; and alcohol use, suicide ideation, presenteeism and absenteeism.

\section{Stress}

Analysis of the 2,053 valid responses showed that more than half (54.9\%) of dentists reported currently experiencing high job stress and when looked at by field of practice, GDPs reported the highest levels of stress.

Worryingly, nearly half of the dentists in this sample (43.8\%) said they could not cope with the level of stress in their job.

This varied by field of practice with GDPs exhibiting the highest scores, indicating they could not cope with the level of stress, followed by community dentists. Both were significantly higher than hospital dentists and dentists working in another field of practice.

The most common sources of stress related to threat of complaints/ litigation (79\%), dissatisfied patients (75.1\%), concern about the GDC (72.8\%), followed by work pressure such as NHS targets (72.4\%), running behind schedule (64.9\%), NHS work (63.2\%) and difficult patients (61.2\%).

\footnotetext{
Alcohol

One way of coping with stress appeared to be with higher than normal consumption of alcohol, the researchers found. For all respondents, $85.6 \%$ had drunk alcohol in the last 12 months and of those, around a fifth (22.1\%) reported drinking four times or more $\circlearrowleft$ every week, with $5.6 \%$ drinking every day.
}

This compares to $9.6 \%$ of people (aged 16 and over) in Great Britain in 2016 who report drinking on five or more days a week. However, when the researchers compared respondents who said they had high stress in their jobs and those who did not, there was no significant difference in the self-reported frequency of alcohol consumption between the two groups.

Suicide

A deadly consequence of high levels of stress was evident in the survey responses as the analysis showed that $17.6 \%$ of all respondents had seriously thought about committing suicide at some point in their lives, which is comparable to a recent survey that found that $20.6 \%$ of the general population had reported suicidal thoughts at one point in their life.

However, the BDA researchers found that dentists displayed much higher levels of suicidal thought than the general population. Of those who had said they had thought about suicide, $57.7 \%$ had thought about committing suicide in the last 12 months.

This equates to nearly $10 \%$ of respondents, and is higher than the general population figure of 5.4\% reported for 16-74-year-olds living in England, and slightly higher than the global figure of $9.2 \%$ found in other research.

The authors concluded: 'The high levels of self-reported stress, burnout and psychological distress found in this study are a serious concern to the profession.

'Although GDPs seem to be particularly affected, this research shows that all fields of dentistry exhibit elevated levels compared to the general population, other professions including veterinary surgeons, GPs or dentists in the same field of practice working in other countries. Therefore, highlighting that this is a universal problem for dentists working in the UK.

One of the authors, Dr Vicki Collin, BDA Research Analyst, said it was evident that aspects relating to regulation and the fear of litigation were key concerns among UK dentists, adding: 'Implementing regulatory changes by reducing the amount of regulatory burden could go a long way in reducing the amount of stress experienced by dentists.

'Other environmental factors such as the NHS contract in England and Wales was also a key source of stress (among GDPs working in the NHS), especially pressure to attain UDA targets. A reformed contract that is not so target driven and focuses on prevention would be welcomed.

'Increasing the support available to dentists who are experiencing high stress or burnout so that all dentists have a dedicated, fully funded confidential support service, similar to what GPs have access to would be beneficial.' 